\title{
Aleitamento materno exclusivo e adiposidade
}

\author{
Exclusive breastfeeding and adiposity
}

José Fernando V. N. Moraes ${ }^{1}$, Rodolfo Giugliano ${ }^{2}$

\section{RESUMO}

Objetivo: Associar o tempo de amamentação exclusiva da criança à adiposidade central e periférica, por meio do índice de massa corporal, dos perímetros da cintura e do braço, e das dobras cutâneas triciptal, subescapular e a somatória destas em pré-escolares.

Métodos: Pesquisa de delineamento transversal, em que 134 pré-escolares entre três e cinco anos de idade de uma escola particular de Brasília, DF, foram avaliados quanto a: massa corporal, estatura, perímetros do braço e da cintura, dobras cutâneas triciptal e subescapular. Os pais das crianças responderam a um questionário sobre tempo de amamentação. O diagnóstico de sobrepeso e obesidade foi realizado de acordo com a classificação da Organização Mundial da Saúde para o índice de massa corporal por idade.

Resultados: As meninas tiveram maior concentração adiposa na dobra cutânea triciptal $(p=0,001)$, subescapular $(p=0,044)$ e na somatória destas $(p=0,003)$ em relação aos meninos. A prevalência de sobrepeso e obesidade foi similar nos dois sexos (25,4\% nos meninos e 22,6\% nas meninas), assim como o tempo médio de amamentação exclusiva (4,3 meses para meninos e 4,6 meses para meninas). Notou-se correlação inversa significativa entre tempo de amamentação exclusiva e perímetro da cintura $(\mathrm{r}=-0,166 ; p=0,05)$. As demais variáveis também mostraram tendência de correlação inversa com o tempo de aleitamento materno exclusivo, porém sem valores significativos.

Conclusões: A associação inversa entre o tempo de amamentação e o perímetro da cintura mostra um possível efeito do aleitamento materno sobre a distribuição de gordura corporal no pré-escolar.

Palavras-chave: aleitamento materno; adiposidade; obesidade abdominal; pré-escolar.

Instituição: Universidade Católica de Brasília (UCB), Brasília, DF, Brasil ${ }^{1}$ Mestre em Educação Física pela UCB; Professor Assistente da Universidade Federal do Vale do São Francisco (Univasf), Petrolina, PE, Brasil 2Doutor em Nutrição Humana pela University of London, Inglaterra; Professor Titular de Pediatria da UCB, Brasília, DF, Brasil

\section{ABSTRACT}

Objective: To associate exclusive breastfeeding with central and peripheral adiposity measured by body mass index, waist and arm circumferences, triceps and subscapular skinfolds and their sum in preschool children.

Methods: This cross-sectional study enrolled 134 preschool children aged 3-5 years from a private school in Brasília, Brazil. All children had their body weight, height, waist and arm circumferences, and triceps and subscapular skinfolds measured. Children's parents answered a questionnaire about breastfeeding duration. Overweight and obesity were diagnosed based on the World Health Organization's classification for the body mass index for age.

Results: Girls had higher adiposity in the triceps skinfold $(p=0.001)$, subscapular skinfold $(p=0.044)$ and in their sum $(p=0.003)$, when compared to boys. Prevalence of overweight and obesity was similar between genders (25.4\% for boys and $22.6 \%$ for girls), as it was exclusive breastfeeding (4.3 months for boys and 4.6 months for girls). A significant inverse correlation was found only between exclusive breastfeeding and waist circumference $(r=-0.166 ; p=0.05)$. Other anthropometric variables showed a trend to present an inverse correlation with exclusive breastfeeding, but lacked statistical significance.

Conclusions: The significant inverse association between exclusive breastfeeding and waist circumference indicates a possible effect of breastfeeding in body fat distribution in preschool children.

Key-words: breastfeeding; adiposity; obesity, abdominal; child, preschool.

Endereço para correspondência:

José Fernando V. N. Moraes

Avenida José de Sá Mariçola, S/N - Centro

CEP 56304-917 - Petrolina/PE

E-mail: josefernandomoraes@gmail.com

Conflito de interesse: nada a declarar

Recebido em: 1/4/2010

Aprovado em: 13/10/2010 


\section{Introdução}

O aumento da obesidade infantil nas últimas duas décadas tem levantado uma série de hipóteses sobre os motivos do desencadeamento desse processo. Setian $e t$ a ${ }^{(1)}$ relataram que o desenvolvimento da obesidade poderia advir de um desequilíbrio entre a ingestão calórica e o gasto energético ou ser determinado por fatores genéticos, fisiopatológicos (endócrino-metabólicos), ambientais (prática alimentar e atividade física) e psicológicos.

Nesse sentido, diversos estudos têm procurado relacionar a obesidade com variáveis ambientais influentes na vida das crianças. Entre estas, encontra-se o aleitamento materno, que, além de melhorar o desenvolvimento neurológico, visual, psicossocial e proteger contra várias morbidades ${ }^{(2)}$, é relatado como fator protetor para o desenvolvimento de sobrepeso e obesidade em inúmeros estudos ${ }^{(3-7)}$.

O diagnóstico de sobrepeso e obesidade nos primeiros anos de vida pode auxiliar na prevenção e no combate ao excesso de peso, evitando o desenvolvimento de doenças cardiovasculares e degenerativas como aterosclerose, intolerância à glicose, diabetes melito tipo 2, dislipidemia e hipertensão $\operatorname{arterial}^{(8)}$. A divulgação de um novo padrão de crescimento pela Organização Mundial da Saúde (OMS), que, além do peso e da estatura, inclui indicadores como o perímetro do braço e dobras cutâneas triciptal e subescapular ${ }^{(9)}$, facilita o diagnóstico e a avaliação da adiposidade em crianças.

Dessa forma, o presente estudo propõe a hipótese de que as crianças amamentadas de forma exclusiva por mais tempo possuiriam valores mais baixos nos indicadores antropométricos de adiposidade, tendo em vista o fator protetor do leite materno no desenvolvimento de sobrepeso e obesidade ${ }^{(3-7)}$. Assim, o objetivo do estudo foi verificar possíveis associações entre o tempo de amamentação exclusiva da criança, o índice de massa corporal (IMC) e a adiposidade central e periférica, por meio dos perímetros da cintura e do braço, das dobras cutâneas triciptal e subescapular, e pela somatória destas em crianças pré-escolares de uma escola particular de Brasília, DF.

\section{Método}

Foram avaliados 134 pré-escolares entre três e cinco anos de idade estudantes do Centro Educacional Católico de Brasília (CECB), escola particular de Brasília com cerca de 3.500 alunos, sendo 330 entre três e cinco anos, predominantemente de classe média e média alta.
Em apenas um encontro (estudo transversal), as crianças foram avaliadas quanto à sua massa corporal, estatura, perímetro da cintura, perímetro do braço e dobras cutâneas triciptal e subescapular do lado esquerdo, segundo as orientações propostas na elaboração do padrão da $\mathrm{OMS}^{(10)}$. Todas as crianças na faixa etária entre três e cinco anos foram convidadas a participar do estudo, com a pré-condição de os pais preencherem um questionário com perguntas sobre amamentação (exclusiva e complementar) e assinarem um termo de consentimento livre e esclarecido. A pesquisa teve aprovação do Comitê de Ética para Pesquisa da Universidade Católica de Brasília (UCB).

Para a medida da massa corporal, foi utilizada uma balança Filizola (São Paulo, Brasil), com $100 \mathrm{~g}$ de precisão. A estatura foi avaliada com estadiômetro Gofeka (Santa Catarina, Brasil), cuja precisão é de $0,1 \mathrm{~cm}$. Os perímetros do braço e da cintura foram medidos com fita antropométrica inextensível Cescorf ${ }^{\circledast}$ (Rio Grande do Sul, Brasil) com precisão de $0,1 \mathrm{~cm}$. A medida do perímetro da cintura foi realizada com as crianças na posição ereta, no ponto médio entre a última costela e a crista ilíaca. As dobras cutâneas triciptal e subescapular foram medidas com adipômetro Lange (Maryland, EUA) com precisão de $0,5 \mathrm{~mm}$. A dobra triciptal foi medida no ponto médio entre o olécrano e o acrômio do braço esquerdo e a subescapular, logo abaixo do ângulo inferior da escápula esquerda.

As crianças foram diagnosticadas como magras, eutróficas, com sobrepeso ou obesas, de acordo com os escores $\mathrm{Z}$ preconizados pela $\mathrm{OMS}^{(11)}$ e adotados pelo Ministério da Saúde, por meio da utilização dos programas Anthro e Anthroplus.

O cálculo amostral foi realizado a partir da equação para estimar amostras de uma população finita ${ }^{(12)}$, sendo o nível de confiança estipulado em $95 \%$, que corresponde ao valor 1,96 expresso em números de desvios padrão. A porcentagem na qual o fenômeno ocorre foi fixada em $16,6 \%$, visto que as crianças incluídas no estudo poderiam ser exclusivamente amamentadas por um mês até os seis meses de vida; a população total da escola analisada era de 330 alunos de três a cinco anos, e o erro amostral foi estipulado em $5 \%$. De acordo com esse cálculo, a amostra necessária para a realização do estudo seria de 130 crianças.

Para comparações entre as medidas antropométricas e a prevalência de sobrepeso e obesidade, de acordo com o sexo, aplicou-se o teste $t$ para amostras independentes. A correlação de Pearson foi empregada para análise das relações entre o tempo de amamentação e as variáveis antropométricas. 
Utilizou-se o programa Statistical Package for the Social Sciences $^{\circledR}$ (SPSS) for Windows 15.0, e o nível de significância adotado foi $p<0,05$. O cálculo do poder foi realizado com o auxílio do programa $G^{*}$ Power 3.0.10 e alcançou valores de 0,81 para o teste de comparação de médias e 0,95 para o teste de correlação.

\section{Resultados}

Das 134 crianças avaliadas, 71 eram do sexo feminino; $20,1 \%(\mathrm{n}=27)$ tinham três anos de idade, $37,3 \%(\mathrm{n}=50)$ tinham quatro anos e $42,5 \%(n=57)$ tinham cinco anos.

A Tabela 1 mostra as características gerais das crianças. $\mathrm{O}$ teste $t$ para amostras independentes indicou diferença significante entre os sexos para a dobra cutânea triciptal $(p=0,001)$, dobra cutânea subescapular $(p=0,044)$ e somatório das duas dobras cutâneas $(p=0,003)$, havendo maior adiposidade nas meninas.
O sobrepeso e a obesidade foram identificados em $23,8 \%(\mathrm{n}=32)$ dos pré-escolares, com 25,4\% ( $\mathrm{n}=16)$ ocorrendo em meninos e $22,6 \%(n=16)$ em meninas. A magreza foi detectada em apenas uma criança $(0,7 \%)$. Não foram observadas diferenças significantes na frequência de excesso de peso entre os sexos e nas diferentes faixas etárias (Tabela 2).

O tempo médio de amamentação foi de 4,5 $\pm 1,6$ meses, sem diferenças entre os sexos. A frequência de excesso de peso nas crianças amamentadas exclusivamente até o sexto mês foi de $21,2 \%$, enquanto que naquelas com amamentação exclusiva até o segundo mês, a frequência foi de $26,7 \%$. A correlação de Pearson entre o tempo de amamentação das crianças e as variáveis antropométricas avaliadas revelou associação inversa com todas as variáveis. A correlação foi significativa quanto ao perímetro da cintura $(\mathrm{r}=-0,166 ; p=0,05)$, como pode ser visto na Tabela 3 e na Figura 1.

Tabela 1 - Medidas antropométricas dos pré-escolares divididos por sexo (valores médios e desvio padrão)

\begin{tabular}{lrrr}
\hline & \multicolumn{1}{c}{ Meninas } & \multicolumn{1}{c}{ Meninos } & \multicolumn{1}{c}{ Total } \\
\hline Massa corporal $(\mathrm{kg})$ & $17,8(3,3)$ & $18,5(3,0)$ & $18,1(3,2)$ \\
Estatura $(\mathrm{cm})$ & $105,3(7,5)$ & $107,1(6,9)$ & $106,2(7,3)$ \\
IMC $\left(\mathrm{kg} / \mathrm{cm}^{2}\right)$ & $15,9(1,5)$ & $16,0(1,7)$ & $16,0(1,6)$ \\
Perímetro do braço $(\mathrm{cm})$ & $16,8(1,6)$ & $16,7(1,7)$ & $16,8(1,7)$ \\
Perímetro da cintura $(\mathrm{cm})$ & $51,8(4,9)$ & $52,5(4,3)$ & $52,1(4,6)$ \\
DCT $(\mathrm{mm})$ & $9,0(2,4)^{*}$ & $7,7(1,8)$ & $8,4(2,3)$ \\
DCS $(\mathrm{mm})$ & $5,9(2,0)^{*}$ & $5,3(1,8)$ & $5,6(1,9)$ \\
$\Sigma$ DC $(\mathrm{mm})$ & $15,0(4,1)^{*}$ & $13,0(4,0)$ & $14,1(3,9)$ \\
\hline
\end{tabular}

${ }^{*} p \leq 0,05$; IMC: índice de massa corporal; DCT: dobra cutânea triciptal; DCS: dobra cutânea subescapular; $\Sigma$ DC: somatório das dobras cutâneas triciptal e subescapular.

Tabela 2 - Frequência de pré-escolares magros, eutróficos, sobrepesados e obesos com base no índice de massa corporal por idade, segundo a classificação da Organização Mundial da Saúde ${ }^{(11)}$

\begin{tabular}{lccc}
\hline & Meninas (\%) & Meninos (\%) & Total (\%) \\
\hline Magreza & - & $1(1,6)$ & $1(0,7)$ \\
Eutrófico & $55(77,5)$ & $46(73,0)$ & $101(75,4)$ \\
Risco de sobrepeso & $7(9,9)$ & $8(12,7)$ & $15(11,2)$ \\
Sobrepeso & $8(11,3)$ & $6(9,5)$ & $14(10,4)$ \\
Obesidade & - & $2(3,2)$ & $2(1,5)$ \\
Obesidade grave & $1(1,4)$ & - & $1(0,7)$ \\
\hline
\end{tabular}

Tabela 3 - Correlação de Pearson entre tempo de amamentação exclusiva (zero a seis meses) e variáveis antropométricas

\begin{tabular}{lcccccc}
\hline & IMC & PC & PB & DCT & DCS & $\boldsymbol{\Sigma}$ DC \\
\hline Amamentação & $-0,088$ & $-0,166^{*}$ & $-0,046$ & $-0,074$ & $-0,101$ & $-0,091$ \\
\hline
\end{tabular}

${ }^{*} p \leq 0,05$; IMC: índice de massa corporal; PC: perímetro da cintura; PB: perímetro do braço; DCT: dobra cutânea triciptal; DCS: dobra cutânea subescapular; $\Sigma$ DC: somatório das dobras cutâneas triciptal e subescapular. 


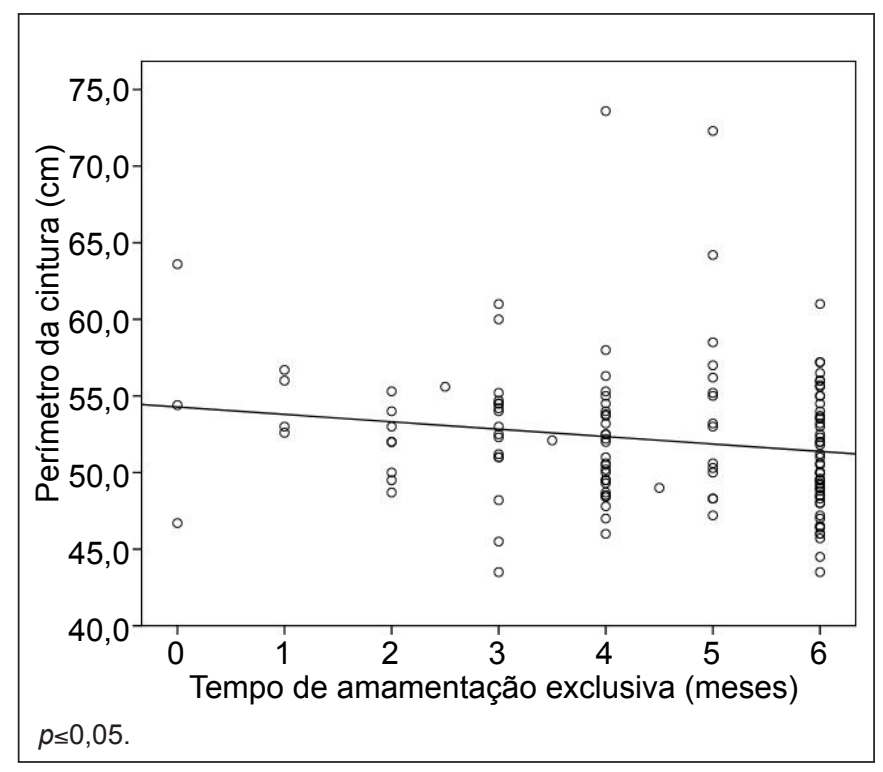

Figura 1 - Dispersão entre o perímetro da cintura e o tempo de amamentação exclusiva.

\section{Discussão}

Inúmeros autores têm relacionado o tempo de amamentação exclusiva e o desenvolvimento de sobrepeso e obesidade. Destacam-se os estudos de Siqueira e Monteiro(3), Victora et al $^{(4)}$, Koletzko et $a l^{(5)}$, Harder et $a l^{(6)}$, Shields et $a l^{(7)}$, von Kries et a $l^{(13)}$ e Gillman et al ${ }^{(14)}$, que constataram um efeito dependente entre a duração do aleitamento materno e a incidência de sobrepeso e obesidade em crianças e adolescentes. Bergmann $e a^{(15)} \mathrm{ob}-$ servaram valores estatisticamente maiores das dobras cutâneas triciptal e subescapular em crianças aleitadas artificialmente.

Estudos de revisão e meta-análise também têm mostrado evidências de que o prolongamento da amamentação exclusiva está associado a menor prevalência de obesidade e a um IMC mais baixo, em comparação às crianças e adolescentes que consumiram fórmulas lácteas ${ }^{(2,16,17)}$. Owen et al ${ }^{(18)}$ associaram o aleitamento materno com menores concentrações de insulina sérica, glicemia sanguínea pré-prandial, insulina pré e pós-prandial e um risco cerca de $40 \%$ menor de desenvolver diabetes melito tipo 2. Martin et al ${ }^{(19)}$ relataram uma associação inversa entre amamentação e pressão arterial elevada.

\section{Referências bibliográficas}

1. Setian N, Damiani D, Della Manna T, Ditchchekenian V, Cardoso AL. Obesidade na criança e no adolescente: buscando caminhos desde o nascimento. São Paulo: Roca; 2007

2. Owen CG, Martin RM, Whincup PH, Davey-Smith G, Gillman MW, Cook
Por outro lado, a literatura também traz estudos que não encontram associação entre amamentação e excesso de peso. Li et al ${ }^{(20)}$ não observaram efeito protetor do aleitamento materno no desenvolvimento da obesidade. Kramer et al ${ }^{(21)}$ não constataram efeitos benéficos da amamentação exclusiva prolongada na pressão arterial, estatura, IMC, perímetro da cintura, dobra cutânea triciptal e subescapular. Burdette $e t a^{(22)}$ não encontraram resultados estatisticamente significativos ao comparar adiposidade corporal, por meio de absortometria radiológica de dupla energia (DEXA), com tempo de amamentação ou introdução de alimentação complementar.

Uma limitação inerente ao presente estudo pode ter sido a quantificação do tempo de aleitamento materno exclusivo por meio da utilização de questionários, o que pode ter levado a um viés de esquecimento por parte dos pais. Porém, outros estudos disponíveis na literatura relatam a coleta desses dados por meio da utilização do mesmo instrumento ${ }^{(14,20)}$. Além disso, o delineamento transversal desta pesquisa só permite avaliar o grau de adiposidade em um momento da vida das crianças, tornando necessária a realização de estudos longitudinais para melhor acompanhamento do crescimento e desenvolvimento dos pacientes analisados.

$\mathrm{O}$ presente estudo evidenciou tendência a uma menor ocorrência de sobrepeso e obesidade em pré-escolares amamentados de forma exclusiva até o sexto mês de vida. Além disso, observou-se uma correlação inversa significativa entre perímetro da cintura e tempo de amamentação. Tal resultado também foi observado por Rudnicka et al ${ }^{(23)}$, para os quais indivíduos amamentados por mais de um mês possuíam menor perímetro da cintura, menor relação cintura/quadril e risco $15 \%$ menor de desenvolver obesidade, além de níveis menores de marcadores inflamatórios, quando comparados aos que foram amamentados por menos de 30 dias.

A importância desta observação deve-se ao conhecido efeito da adiposidade visceral como fator predisponente para o desenvolvimento de doenças crônico-degenerativas, como hipertensão arterial, dislipidemia, síndrome metabólica, diabetes melito tipo 2, entre outras ${ }^{(24-29)}$. Tal achado evidencia um possível efeito do aleitamento materno sobre a distribuição de gordura corporal na infância.
DG. The effect of breastfeeding on mean body mass index throughout life: a quantitative review of published and unpublished observational evidence. Am J Clin Nutr 2005;82:1298-307.

3. Siqueira RS, Monteiro CA. Amamentação na infância e obesidade na idade 
escolar em famílias de alto nível socioeconômico. Rev Saude Publica 2007;41:5-12.

4. Victora CG, Barros F, Lima RC, Horta BL, Wells J. Anthropometry and body composition of 18 year old men according to duration of breast feeding: birth cohort study from Brazil. BMJ 2003;327:901.

5. Koletzko B, von Kries R, Closa R, Escribano J, Scaglioni S, Giovannini M et al. Can infant feeding choices modulate later obesity risk? Am J Clin Nutr 2009;89 (Suppl 1):1502S-8S.

6. Harder T, Bergmann R, Kallischnigg G, Plagemann A. Duration of breastfeeding and risk of overweight: a meta-analysis. Am J Epidemiol 2005;162:397-403.

7. Shields L, O'Callaghan M, Williams GM, Najman JM, Bor W. Breastfeeding and obesity at 14 years: a cohort study. J Paediatr Child Health 2006;42:289-96.

8. Almeida CA, Pinho AP, Ricco RG, Elias CP. Circunferência abdominal como indicador de parâmetros clínicos e laboratoriais ligados à obesidade infanto-juvenil: comparação entre duas referências. J Pediatr (Rio J) 2007;83:181-5.

9. de Onis M, Victora CG, Garza C, Frongillo EA Jr, Cole T. A new international growth reference for young children. In: Dasgupta P, Hauspie R, editors. Perspectives in human growth, development and maturation. Dordrecht, The Netherlands: Kluwer; 2001. p. 45-53.

10. de Onis M, Onyango AW, Van den Broeck J, Chumlea WC, Martorell R. Measurement and standardization protocols for anthropometry used in the construction of a new international growth reference. Food Nutr Bull 2004;25 (Suppl 1):S27-36.

11. Onyango AW, de Onis M. WHO childgrowth Standards: training course on child growth assessment. Geneva: World Health Organization; 2008.

12. Triola MF. Introdução à estatística. 9a ed. Rio de Janeiro: LTC; 2005.

13. von Kries R, Koletzko B, Sauerwald T, von Mutius E, Barnert D, Grunert V et al. Breast feeding and obesity: cross sectional study. BMJ 1999;319:147-50.

14. Gillman MW, Rifas-Shiman SL, Camargo CA Jr, Berkley CS, Frazier AL, Rockett HR et al. Risk of overweight among adolescents who were breastfed as infants. JAMA 2001;285:2461-7.

15. Bergmann KE, Bergmann RL, von Kries R, Böhm O, Richter R, Dudenhausen JW et al. Early determinants of childhood overweight and adiposity in a birth cohort study: role of breast-feeding. Int J Obes Relat Metab Disord 2003;27:162-72.

16. Balaban G, Silva GA. Protective effect of breastfeeding against childhood obesity. J Pediatr (Rio J) 2004;80:7-16
17. Martorell R, Stein AD, Schroeder DG. Early nutrition and later adiposity. J Nutr 2001;131:874S-80.

18. Owen CG, Martin RM, Whincup PH, Smith GD, Cook DG. Does breastfeeding influence risk of type 2 diabetes in later life? A quantitative analysis of published evidence. Am J Clin Nutr 2006;84:1043-54.

19. Martin RM, Gunnell D, Smith GD. Breastfeeding in infancy and blood pressure in later life: systematic review and meta-analysis. Am J Epidemiol 2005;161:15-26.

20. Li L, Parsons TJ, Power C. Breast feeding and obesity in childhood: cross sectional study. BMJ 2003;327:904-5.

21. Kramer MS, Matush L, Vanilovich I, Platt RW, Bogdanovich N, Sevkovskaya Z et al. Effects of prolonged and exclusive breastfeeding on child height, weight, adiposity, and blood pressure at age $6.5 \mathrm{y}$ : evidence from a large randomized trial. Am J Clin Nutr 2007;86:1717-21.

22. Burdette HL, Whitaker RC, Hall WC, Daniels SR. Breastfeeding, introduction of complementary foods, and adiposity at $5 \mathrm{y}$ of age. Am J Clin Nutr 2006;83:550-8.

23. Rudnicka AR, Owen CG, Strachan DP. The effect of breastfeeding on cardiorespiratory risk factors in adult life. Pediatrics 2007;119:e1107-15.

24. Katzmarzyk PT, Srinivasan SR, Chen W, Malina RM, Bouchard C, Berenson GS. Body mass index, waist circumference, and clustering of cardiovascular disease risk factors in a biracial sample of children and adolescents. Pediatrics 2004;114:e198-205.

25. Lee S, Bacha F, Arslanian SA. Waist circumference, blood pressure, and lipid components of the metabolic syndrome. J Pediatr 2006;149:809-16.

26. Montañés EC, Geraud AA, Sardiña NG, Bustos CL. Waist circumference, dyslipidemia and hypertension in prepubertal children. An Pediatr (Barc) 2007;67:44-50.

27. Moreno LA, Pineda I, Rodríguez G, Fleta J, Sarría A, Bueno M. Waist circumference for the screening of the metabolic syndrome in children. Acta Paediatr 2002;91:1307-12.

28. Freedman DS, Serdula MK, Srinivasan SR, Berenson GS. Relation of circumferences and skinfold thicknesses to lipid and insulin concentrations in children and adolescents: the Bogalusa Heart Study. Am J Clin Nutr 1999;69:308-17.

29. lampolski MN, Souza FI, Sarni RO. Influência do índice de massa corporal e da circunferência abdominal na pressão arterial sistêmica de crianças. Rev Paul Pediatr 2010;28:181-7. 